\title{
Surface Effect Contributes to Small Structures' Surprising Strength
}

\author{
Experiments that compress cubes containing gold nanowires suggest that \\ a previously overlooked feature may help explain the surprising strength \\ of tiny objects.
}

By Dan Garisto

S tructures smaller than a micrometer can become, paradoxically, quite strong. New experiments suggest that a previously overlooked material feature known as a surface triple junction (STJ) may be an important factor in nanomaterial strength [1]. An STJ is a line on a surface where the crystalline grains of a material meet, analogous to the boundaries between planks in a hardwood floor. STJs are common in materials, such as thin film semiconductors, so their strength characteristics could become an important consideration in the design of nanoscale devices. However, some experts have raised questions about the results, suggesting that the STJ effect is not the only possible interpretation of the data.
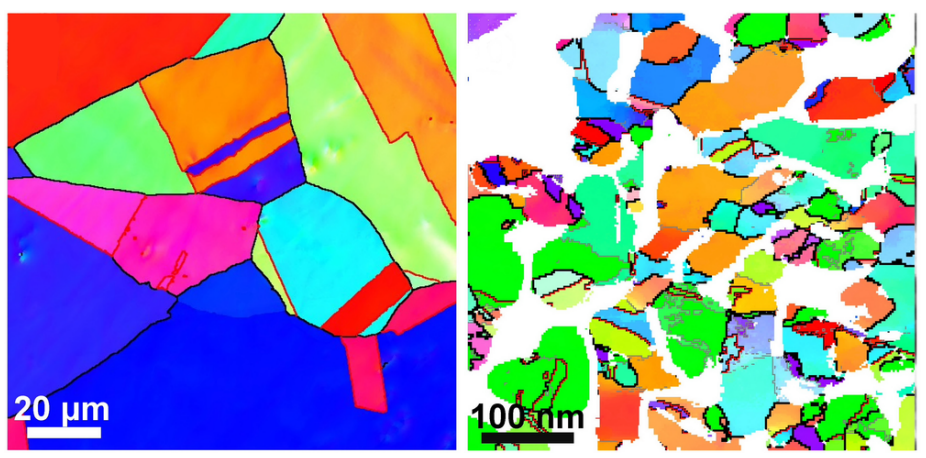

Grains galore. False color maps of grains-individual crystallites-in coarse-grained (left) and fine-grained (right) nanoporous gold.

Credit: Y.-Y. Zhang et al. [1]
Over the past few decades, precision measurements and advances in theory have allowed researchers to explain some mechanisms responsible for the surprising strength of small things. A crystal can be weakened by dislocations-abrupt shifts of atoms in a lattice, similar to shifted blocks in a Jenga tower. However, depending on their number and configuration, dislocations can also strengthen a crystal by preventing it from yielding in response to compression.

Because dislocations can have an important influence on strength, researchers have focused on places where they collect. For example, dislocations often form at grain boundaries, which are the 2D interfaces that separate the grains-regions with different crystal structure orientations-within a crystalline material. STJs are the lines formed by grain boundaries at the surface of a material, and no one has carefully studied the effects of STJs on material strength.

"It's very difficult to separate the effects" of STJs from those of grain boundaries and non-STJ surface phenomena, says Hai-Jun Jin, of the Institute of Metal Research in China. But Jin and his colleagues have developed techniques that they say have allowed them to distinguish the STJ effect.

The researchers used millimeter-sized cubes of nanoporous gold-sponge-like structures made of roughly one trillion gold nanowires, or "ligaments." They studied two types of samples: coarse-grained, with few grain boundaries, and fine-grained, 
with many grain boundaries. The team measured the samples' strengths by compressing them and observing their deformation. "Strength" in these experiments is the force needed to steadily deform the sample, in the so-called plastic regime, where its shape doesn't spring back. They performed the compression experiments in an electrolyte bath that produced an oxide on the gold surfaces when a high voltage was applied.

The researchers measured the difference in strength between the oxidized and clean states for both coarse- and fine-grained samples with a range of ligament widths. (Thinner ligaments were expected to amplify surface effects because of their higher surface-to-volume ratio.) For samples with the thinnest ligaments, surface oxidation had a much greater effect on the strength of fine-grained samples than on the strength of coarse-grained ones. Fine-grained samples had more grain boundaries and more STJs than coarse-grained ones, but grain boundaries are internal and unaffected by the surface treatment. So Jin and his colleagues concluded that only STJs could be responsible for the difference between the two sample types.

In fact, the data suggested that the STJ effect could be a dominant factor for material strength at sub-100-nanometer scales, based on the effect of ligament width on the strength differences. The team proposes that STJs might be influencing material strength because they create a place for dislocations to grow.

One challenge in the analysis was that the team could only measure the strength of the sample, but not that of individual ligaments. Without more understanding of the relationship between macroscopic measurements and the ligament properties, they were unable to determine whether the STJs made individual ligaments stronger or weaker.

Materials scientists Cynthia Volkert of the University of Göttingen, Germany, and David Srolovitz of the City University of Hong Kong both say that there might be additional mechanisms at play besides dislocation, so there may be more than just STJs involved. Srolovitz suggests that a process called grain-boundary sliding could also be affecting material strength in these experiments. In response, Jin says grain-boundary sliding was the first mechanism that came to mind when he saw the data, but he and his colleagues could not find conclusive evidence for it.

Materials scientist Jörg Weismüller of the Hamburg University of Technology in Germany says the work is important. "The observation must have been astonishing to the authors, that they saw such a strong and systematic effect," he says. "I was particularly impressed by the fact that they came up with such an elegant explanation."

Dan Garisto is a freelance science writer based in New York.

\section{REFERENCES}

1. Y.-Y. Zhang et al., "Surface triple junctions govern the strength of a nanoscale solid,” Phys. Rev. Lett. 126, 235501 (2021). 